Año LXXXI. urtea $278-2020$

Septiembre-diciembre Iraila-abendua

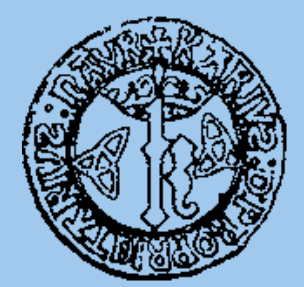

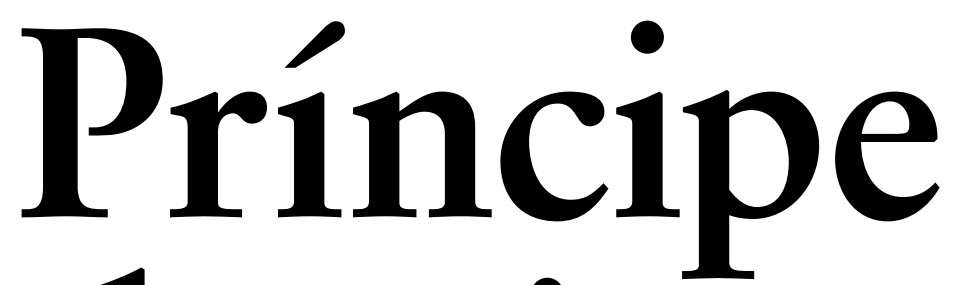

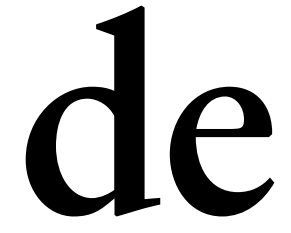
Viai iana

SEPARATA

\section{Gustav Henningsen: del antropólogo al historiador (pasando por archivero)}

Juan Ignacio PULIDO SERRANO 


\section{Sumario / Aurkibidea}

\section{Príncipe de Viana}

Año LXXXI - n. ${ }^{\circ} 278$ - septiembre-diciembre de 2020

LXXXI. urtea - 278. zk. - 2020ko iraila-abendua

\section{GUSTAV HENNINGSEN / MARISA REY-HENNINGSEN}

Homenaje / Omenaldia

Ignacio Panizo (coord./koord.)

Vol. I. lib.

Preámbulo / Hitzaurrea

Rebeca Esnaola Bermejo

Presentación / Aurkezpena

$\underline{\text { Ignacio Panizo Santos }}$

\section{TRAYECTORIA VITAL E INTELECTUAL DE GUSTAV HENNINGSEN \\ Y MARISA REY-HENNINGSEN / \\ GUSTAV HENNINGSENEN ETA MARISA REY-HENNINGSENEN \\ BIZITZA ETA IBILBIDE INTELEKTUALA}

Autobiografía de Gustav Henningsen

Autobibliografía de Gustav Henningsen

Bibliografía de Marisa Rey-Henningsen

Transcribiendo a Gustav y Marisa

Candela M. Camiño López

Gustav Henningsen, un encendido asombro ante la realidad

Mikel Azurmendi Inchausti

El danés peligroso. Semblante humano de Gustav Henningsen.

Evocación a cuatro manos

Jean Pierre Dedieu, Gunnar W. Knutsen

«Un danés peligroso» en los fondos del Archivo Histórico Nacional

Jaime Contreras Contreras 


\section{Sumario / Aurkibidea}

Gustav Henningsen: del antropólogo al historiador (pasando por archivero)

Juan Ignacio Pulido Serrano

Gustav Henningsen y Marisa Rey-Henningsen, folcloristas daneses en Galicia, 1965-1977 (entre magnetófonos y cuentos matriarcales)

José Manuel Pedrosa Bartolomé

Marisa Rey-Henningsen y el arte de la traducción de la literatura danesa José Luis Garrosa Gude

OBRA DISPERSA DE GUSTAV HENNINGSEN /

GUSTAV HENNINGSENEN OBRA BARREIATUA

Los documentos de Alonso de Salazar Frías. Una polémica sobre la brujería en España, 1610-1614

Gustav Henningsen

De la caza de brujas al culto de brujas

Gustav Henningsen

Archivos e historiografía de la Inquisición española

Gustav Henningsen

El síndrome de brujería infantil: el abuso infantil satánico contemporáneo

y los procesos por brujería infantil de antaño

Gustav Henningsen

999

La brujería y la Inquisición

Gustav Henningsen

Enciclopedia de la brujería

Gustav Henningsen

El vuelo de las brujas y los inquisidores españoles o cómo explicar lo imposible

Gustav Henningsen

Currículums

Analytic Summary

Normas para la presentación de originales / Idazlanak aurkezteko arauak /

Rules for the submission of originals 


\section{Gustav Henningsen: del antropólogo al historiador (pasando por archivero)}

Gustav Henningsen: antropologo izatetik historialari izatera (tartean artxibozain izanda)

Gustav Henningsen: from the anthropologist to the historian (as well as the archivist)

Juan Ignacio Pulido Serrano

Profesor de la Universidad de Alcalá de Henares

jignacio.pulido@uah.es

DOI: https://doi.org/10.35462/pv.278.9 


\section{RESUMEN}

En este trabajo se expone la evolución experimentada por la investigación de Gustav Henningsen en los campos de la antropología, de la historia y de la archivística. Se presenta también el sentido de su obra, con las principales aportaciones que ha realizado a la historia social y cultural de Europa en la Edad Moderna, en temáticas como son las prácticas y creencias mágicas de las sociedades del pasado. Entre sus aportaciones se señalan sus descubrimientos y estudios de fondos procedentes de los archivos de la Inquisición española, los métodos de trabajo novedosos que aplicó sobre ellos y la creación de herramientas para el análisis que han sido utilizadas posteriormente.

Palabras clave: antropología; archivística; Inquisición; cultura popular; brujería.

\section{LABURPENA}

Lan honetan adierazten da Gustav Henningsenen ikerlanak zer bilakaera izan zuen antropologia, historia eta artxibozaintzaren alorretan. Haren obraren zentzua ere azaltzen da; baita zer ekarpen nagusi egin dituen ere Aro Modernoko Europaren historia sozial eta kulturalean; besteak beste, antzinako gizarteen praktika eta sineste magikoen esparruari dagokionez. Haren ekarpenetako batzuk dira Espainiako Inkisizioaren artxiboetatik datozen funts batzuk aurkitu eta aztertzea, haiei lan-metodo berritzaileak aplikatzea eta gerora erabili diren analisi-tresnak sortzea.

Gako hitzak: antropologia; artxibozaintza; Inkisizioa; herri-kultura; sorginkeria.

\section{ABSTRACT}

This paper sets out the development of Gustav Henningsen's research in the fields of anthropology, history and archival science. It also summarises the meaning of his work as a whole, as well as the main contributions he has made to knowledge of various topics in the social and cultural history of Europe in the modern age, such as the magical practices and beliefs of the societies of the past. These contributions include his discoveries and studies of collections from the archives of the Spanish Inquisition, the innovative working methods he applied to them and the creation of tools for analysis that have been used by scholars to achieve outstanding results in the production of knowledge.

Keywords: Anthropology; Archives; Inquisition; Popular culture; Witchcraft. 
1. INTRODUCCIÓN. 2. LA PRIMERA ANDADURA: EL ANTROPÓLOGO EN BUSCA DEL FOLKLORE NÓRDICO. 3. EL ANTROPÓLOGO EN BUSCA DE LA HISTORIA (Y DE UN MÉTODO DE TRABAJO). 4. EL ARCHIVERO: FUENTES Y MÉTODOS PARA LA HISTORIA. 5. EL ABOGADO DE LAS BRUJAS. 6. ALONSO DE SALAZAR FRÍAS, INQUISIDOR Y HUMANISTA. 7. DE LAS FUENTES Y MÉTODOS DE ANÁLISIS EN LOS ESTUDIOS INQUISITORIALES. 8. LISTA DE REFERENCIAS.

\section{INTRODUCCIÓN}

Si hubiera que señalar el rasgo principal que ha caracterizado la investigación que Gustav Henningsen ha desarrollado durante más de medio siglo, sin lugar a dudas destacaría su constante esfuerzo por poner en relación la antropología con la historia. Y a esto añadiría que, para conseguir tal combinación, ha sido fundamental el trabajo que ha realizado entre los fondos documentales del archivo. Junto a este esfuerzo, al que se ha entregado durante toda su vida profesional, resaltaría además la importancia de los resultados que ha alcanzado, lo que demuestra la eficacia de sus métodos en el intento de ampliar los conocimientos, de forma muy especial, en el campo de la historia.

De tal manera, la primera lección que Henningsen nos deja es el valor de la mirada del antropólogo cuando nos adentramos en el ejercicio propio del historiador y, para que esto sea posible, nos muestra la importancia de las fuentes documentales existentes en los archivos. Solo así, con esto último, el conocimiento de la historia puede descender del estadio de la especulación a la realidad empírica, mostrando el pasado tal como fue y no como tantas veces se imagina. Y el asunto de la brujería y de la brujomanía -objetos centrales de sus indagaciones- es el mejor ejemplo de ello, lo que trataremos de explicar en estas páginas dedicadas al autor.

Como logros concretos de su trabajo indicaremos dos. Primero, siguiendo los pasos de los brujos y de quienes creían en su existencia, Henningsen se enfrentó a unos y otros como lo hace el antropólogo moderno, con los métodos propios aplicados en los «trabajos de campo», en los que se reúnen informaciones vertidas por informan- 
tes vivos, para después someter a análisis todo lo reunido por la observación directa del asunto a estudiar. La dificultad en este punto estaba, como es fácil de entender, en que los informantes de los siglos XVI y XVII dejaron de estar vivos hace demasiado tiempo y no resultaba viable, en consecuencia, preguntarles siguiendo el cuestionario elaborado por el estudioso en su gabinete. Solo la constancia, junto a la experiencia profesional adquirida como archivero, explican que Gustav Henningsen lograra volver a escuchar los relatos detallados de miles de informantes, los cuales fueron narrados a otro antropólogo pesquisidor que le precedió, este de oficio inquisidor y anticipo de los científicos modernos, quien dejó de existir, como sus miles de interrogados, hace cuatrocientos años. Gracias a esto, se nos hace hoy más comprensible el problema de la brujería y del fenómeno de la brujomanía que recorrió Europa durante siglos.

El segundo logro se refiere al conocimiento que nos deja de la historia de la Inquisición y de sus fondos archivísticos, tomados estos como una herramienta disponible y propicia para la investigación de distintos aspectos fundamentales en el campo de la cultura y de las mentalidades que caracterizaron la Europa de la temprana modernidad. La localización de una serie tan valiosa de documentos como son las relaciones de causas y el uso sistemático que Henningsen ensayó con ella demostraron las posibilidades que esta fuente ofrece a la investigación. De su existencia ya se sabía con anterioridad, es cierto. Pero el empleo que él les dio en los años anteriores al despegue informático y a la práctica cuantificadora, advirtió del valor revelador que podía tener esta documentación seriada. Porque esta era una serie documental que, de forma constante y prolongada, se fue elaborando por los tribunales del Santo Oficio durante los siglos XVI y XVII. Y en ella se recogían informaciones sobre los comportamientos y las creencias de los hombres que habitaron en los diversos espacios del imperio español, ya fuera en Europa o en América, allá donde la institución se implantó. Más que poder desvelar el número de las víctimas de la institución, asunto puesto en el centro de la polémica que se venía planteando en torno a la Inquisición, las relaciones de causas se prestaban a que se realizasen con ellas mediciones de distintos problemas de naturaleza social y cultural; y, sobre todo, comparaciones entre la evolución que tales problemas experimentaron en unos territorios y otros. El propio Henningsen lo hizo al comparar la evolución de la brujería y de la hechicería en los distintos ámbitos del imperio español.

El camino recorrido por Gustav Henningsen, desde sus estudios iniciales en la senda de la antropología hasta alcanzar los tocantes a la historia, obedece a una evolución natural, coherente con el dictado de su inquietud intelectual. En su caso, este tránsito cobra un sentido lógico, resultado de su experiencia personal, profesional y académica, y que él mismo ha explicado en diversas ocasiones, dejándolo expresado, también por escrito, con admirable franqueza y honestidad. Basta para entenderlo leer su texto "Andanzas por España de un "inquisidor a la moderna de raza nórdica" ", texto autobiográfico publicado en el año de 2010, después de medio siglo ejercitándose en la investigación. Nada más transparente podría encontrarse en una confesión como la suya (Henningsen, 2010). 


\section{LA PRIMERA ANDADURA: EL ANTROPÓLOGO EN BUSCA DEL FOLKLORE NÓRDICO}

Hay que reconocer en la trayectoria de Gustav Henningsen, que dio comienzo cuando mediaba la década de 1950, una marcada inclinación hacia la aventura, donde la investigación se concibe como búsqueda continuada de lo desconocido, entendido esto como un misterio a desvelar. Investigación, por lo tanto, que le ha exigido una constante y vigorosa acción en la pesquisa, además de un extraordinario tesón, lo que explica la profundidad alcanzada por su trabajo. También se percibe en su empeño una motivación amorosa por el objeto de estudio, y por el mismo estudio inquisitivo, que emana de un amor procesado de manera fiel y también constante. Él mismo se refería a ello cuando explicaba las razones de su personal «trayectoria investigadora», tal como se expresa en términos propios de la burocracia actual. Decía, al respecto, que la suya había sido la historia de varios enamoramientos por diversos temas de estudio que se han ido sucediendo a lo largo de su vida. Visto desde el ahora en el que esto se escribe, todo parece indicar que ha sido solo uno el objeto de su amor, y muchas, sin embargo, las veces que su querencia ha vuelto sobre él.

En 1955, como estudiante universitario, Henningsen se interesaba por las tradiciones populares nórdicas a partir de los contenidos de los cuentos escritos por el literato danés Hans Christians Andersen. Algún tiempo después, un artículo publicado en Norteamérica, que defendía que solo en la cuentística de allá existían cuentos sobre embustes, o de asuntos inverosímiles, le empujó a rastrear esta cuestión concreta en el folklore de su país, descubriéndola y dando cuenta de ella en unos primeros ensayos de juventud (1961 y 1964). Ya se encontraba ahí su inquietud por el desvelamiento de las tradiciones populares que forman el sustrato común de las sociedades europeas, con todas sus variantes posibles. De su encuentro con la lengua española, con poco más de veinte años de edad, había surgido la lectura de los trabajos de Menéndez Pidal sobre el romancero español, y tras ello, su intento de buscar en la tradición danesa los romanceros que nutrieron la cultura de sus compatriotas en los siglos XVI y XVII (1959). La tradición popular: esta fue, según nos confiesa, su «primera amada». Pero es esta problemática, que la historiografía viene denominando como "cultura popular», la que también seguimos encontrando en todos los trabajos que ha realizado hasta la fecha de hoy, pese a que haya sido a través de las diversas vertientes y manifestaciones en las que se nos muestra dicha tradición cultural.

Ciertamente, esta es la constante que se halla en toda su investigación: la búsqueda de los elementos comunes en la cultura popular europea desde la Edad Moderna hasta nuestros días; y, con ello, la detección de las distintas variantes que sobre este mismo basamento se han ido produciendo en las diferentes áreas del continente a lo largo del tiempo. Su intención era encontrar las razones que explican tal diversidad dentro de esta unidad cultural. De tal manera, cuando Henningsen se adentra en los fenómenos de la brujería, de la brujomanía y de la hechicería, este interés sigue presente como cuestión fundamental de su análisis.

En una trayectoria de ejercicio intelectual como es la suya, caracterizada por la constancia -tozudez o «terquedad» dirían sus profesores de Universidad-, estas tradiciones, 
creencias y manifestaciones populares han sido siempre la espina dorsal de sus indagaciones. Con estas inquietudes viajó a España en 1965 decidido a trabajar sobre las creencias en torno a la brujería, y con la intención de compararlas con las que él mismo detectó entre los marineros de las localidades de la isla de Aerö, en Dinamarca. Con la metodología de los antropólogos del momento -y con Evans Pritchard y sus trabajos sobre el pueblo azande de África como principal referencia- quiso ensayar sus propias fórmulas en el norte de España. Saltaba así del estudio entre las gentes marineras de las islas danesas a las que habitaban en las tierras de la cornisa cantábrica española. Y entre estas gentes de un lado y otro, Henningsen quiso poner a prueba algunas fórmulas de comparación en las creencias mágicas que mantenían en torno a la brujería y la hechicería. Las orientaciones de Julio Caro Baroja le llevaron a probar en Galicia sus primeros experimentos en este sentido. Y el método que el antropólogo español venía entonces construyendo - «el estructuralismo histórico», denominación por él utilizada-acabarían por ser determinantes en la propia evolución científica del joven antropólogo danés.

\section{EL ANTROPÓLOGO EN BUSCA DE LA HISTORIA (Y DE UN MÉTODO DE TRABAJO)}

Gustav Henningsen reconoció abiertamente el magisterio que Caro Baroja ejerció sobre él: «En caso de considerarme alumno de alguien, sería de Caro Baroja». Cuando llegó a España en el verano de 1964, con treinta años de edad, decidido a planificar los trabajos de campo para su próxima tesis doctoral, fue primero a discutir su proyecto con él, y después de escuchar sus consejos, aceptó la idea de localizar en Galicia el objeto de su estudio. El libro de Caro Baroja, Las brujas y su mundo, que había aparecido solo unos años atrás, en 1961, fue para él una «especie de Biblia» sobre el tema. Y también fue Caro Baroja -recuerda Henningsen- quien le reafirmó en su consideración crítica de los trabajos del antropólogo Evans Pritchard, con quien Caro había estado en la década anterior en el Instituto de Antropología Social de la Universidad de Oxford. Henningsen ha considerado a Evans Pritchard como uno de sus padres espirituales, y sus trabajos sobre el mundo mágico y las prácticas de hechicería de la tribu azande del centro de África como un modelo de análisis a seguir. Así lo hizo cuando se embarcó en sus investigaciones en las tierras del norte de España.

Del antropólogo funcionalista que fue Pritchard pueden encontrase huellas todavía visibles en las reflexiones con las que Henningsen pone fin a su última edición de $E l$ abogado de las brujas (2010). En el epílogo del libro nos explica que las prácticas brujeriles han venido cumpliendo una función social a lo largo de la historia. Y tal perspectiva funcionalista ya estaba presente en el planteamiento de su primer proyecto de estudio en la Galicia del siglo XX. Así lo explicaba Henningsen cuando se refería a la función que tales supersticiones tenían en beneficio de las economías rurales gallegas, especialmente cuando los hombres carecían de los conocimientos técnicos suficientes con los que afrontar las diversas situaciones críticas que podían padecer. Hablaba así de una forma de «seguro mágico» existente en tales sociedades atrasadas, en las que se recurría a estas prácticas, por ejemplo, para sanar a la vaca o al cerdo enfermo, bienes económicos cuyos daños se achacaban a intervenciones sobrenaturales. 
Pero ciertamente, el modelo de antropólogo a seguir por Henningsen fue Julio Caro Baroja, y este ya había renunciado al método funcionalista cuando en los años cincuenta se dedicó al estudio de las minorías étnicas que poblaron España en el pasado -las de origen musulmán o judío- y de las tradiciones culturales que unos y otros mantuvieron a lo largo del tiempo. Lo que a Julio Caro Baroja le despertaba una fuerte inquietud intelectual era el hecho comprobado de que tales tradiciones permanecieran vivas entre estos grupos minoritarios, a pesar del paso de los siglos y del medio poco favorable, sino hostil, en el que existieron. La secta brujeril, o los linajes de brujos, de existir, respondían también a este mismo fenómeno de continuidad histórica. Esto era precisamente lo que también había despertado el interés intelectual de Gustav Henningsen cuando estudiaba a las gentes marineras de la isla danesa de Aerö. Entre las tradiciones culturales de estos hombres, le atrajo la idea de poder entender las razones que explicaban la larga duración de creencias supersticiosas como eran las referidas a la existencia de brujas, que él había encontrado todavía vigentes entre ciertas personas a las que tuvo la oportunidad de entrevistar.

Fue por esta razón que tras dejar Galicia en el verano de 1964 y volver a encontrase con Julio Caro Baroja en su casa de Bera de Bidasoa, le pidió que le presentara a esos vecinos de la zona que reconocieron haber tenido noticias de la existencia de aquelarres celebrados en sus días. La larga duración del fenómeno es lo que centraba el objeto de su interés y, por lo tanto, su investigación precisaba de ser encaminada hacia el análisis de tales continuidades históricas. Por un lado, de existir la práctica brujeril, resultaba necesario explicar la continuidad en la existencia no solo de tales prácticas sino además de los grupos que las seguían realizando. Él los llamó «linajes de brujos», los cuales ya había reconstruido, con sus respectivas genealogías, en los pueblos de la isla danesa. Por otro lado, era preciso reconstruir la creencia en la brujería en su continuidad histórica, rastrearla a lo largo de la historia, desde el pasado hasta el presente. La perspectiva de su interés tomaba así un marcado enfoque histórico.

Si su viaje desde Dinamarca hasta España estaba organizado en torno al proyecto de llevar a cabo un estudio comparativo de las tradiciones existentes en su país, Irlanda y España, en su viaje de regreso a casa, tras el contacto con Julio Caro Baroja, su objetivo había dado un giro significativo. Su interés por comparar -lanzando una mirada horizontal por la geografía europea que debía poner en relación distintas regiones del continente-, dejó paso a un intento por observar verticalmente la tradición popular en un lugar concreto, para explicar las continuidades en comportamientos y creencias ligados a lo mágico o supersticioso. Caro Baroja le insistió que era Galicia donde mejor podría realizar estos estudios, pues allí era donde podría encontrar vestigios más claros y abundantes de esas creencias procedentes del pasado.

Con esta perspectiva, como un antropólogo lanzado al estudio de la Historia cultural, volvió al año siguiente a Galicia, en 1965. Su idea era hacer allí su tesis sobre esta cuestión histórico-antropológica. Examinaría la historia de la hechicería desde la Edad Moderna hasta el presente y para ello iba pertrechado de los instrumentos de trabajo propios del antropólogo y del historiador. Para lo primero se acogió a la maestría del Carmelo Lisón Tolosana, antropólogo social que andaba haciendo sus trabajos 
de campo por localidades repartidas por las cuatro provincias gallegas. Para lo segundo, se valió del historiador norteamericano Henry Charles Lea, su segundo padre espiritual, como él mismo lo ha denominado. También fue Julio Caro Baroja quien le mostró la importancia de este autor para el estudio de aquellos fenómenos que, como el suyo, andaban a caballo entre la antropología y la historia. Especialmente era de extraordinario valor el uso que había hecho de las fuentes documentales procedentes de los archivos de la Inquisición. Caro Baroja llevaba años sumergido en ellas, para lo que se había guiado también del historiador norteamericano, cuyo libro Historia de la Inquisición de España, publicado en cuatro volúmenes entre 1906 y 1907, era un tesoro en la descripción de fuentes documentales y en las posibilidades que ofrecían para el análisis.

Con esta doble orientación, Henningsen demostró, como nadie lo ha hecho hasta ahora, el extraordinario potencial que tienen estas fuentes de archivo para informar con detalles inimaginables las realidades de un pasado remoto, y que, una vez puestas en la mesa de estudio del antropólogo, permiten el examen de cuestiones que por otro lado serían inalcanzables. Desde el punto de vista de la metodología, esta es la lección más importante que el autor nos ha dejado. La encontramos minuciosamente desplegada en el Abogado de las brujas, libro que, pese a haberse publicado por primera vez hace ya cuarenta años, sigue plenamente vigente. Y, por añadidura, su capacidad inspiradora para futuros trabajos de investigación no ha perdido ni un ápice de su frescura original.

Antes de escribir esta obra, Henningsen se había zambullido durante veinte meses en el ámbito rural gallego, con una grabadora en mano y con las pautas que el antropólogo español, Carmelo Lisón Tolosana, le había mostrado. Inmediatamente después se metió en los fondos del Archivo Histórico Nacional, donde reunió información sobre trescientos procesos contra personas acusadas de brujería y hechicería, y que fueron incoados por el Tribunal de la Inquisición de Santiago de Compostela entre 1565 y 1816 (Henningsen, 1971, p. 63). El hallazgo de este material documental, de enorme valor informativo, le confirmó en su renuncia a la idea inicial de comparar distintos espacios geográficos (Dinamarca, Irlanda y España) y le reafirmó en su nuevo propósito, en una investigación con un marcado sentido histórico: el estudio diacrónico del problema en un espacio geográfico concreto. «Este hallazgo [de los fondos documentales de la Inquisición] me animó -explica- a renunciar a Irlanda y mi proyecto europeo, en favor de un estudio comparativo entre las creencias históricas y actuales de brujería dentro de un mismo ámbito geográfico. Para ello obtuve el visto bueno de mi supervisor» (Henningsen, 2010, p. 429).

\section{EL ARCHIVERO: FUENTES Y MÉTODOS PARA LA HISTORIA}

La documentación que Gustav Henningsen consultó entre los fondos de la Inquisición del Archivo Histórico Nacional entre 1967 y 1968 fueron las llamadas relaciones de causas elaboradas por el Tribunal de Santiago de Compostela, cuyo distrito cubría por entero el antiguo reino gallego, actual Comunidad Autónoma de

876 Príncipe de Viana (PV), 278, iraila-abendua, 2020, 869-887 ISSN: 0032-8472 | ISSN-e: 2530-5824 | ISSN-L: 0032-8472

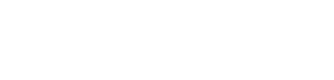


Galicia. En este punto se abrió para él una nueva línea de trabajo que pasaba por el uso exhaustivo de estos fondos archivísticos, ámbito de la investigación en la que ha dejado seguramente su legado más importante. Aquella serie, sobre la que realizó su primer ensayo, centrado en los brujos y hechiceros de Galicia, le había permitido alcanzar unos rendimientos muy altos en poco tiempo, lo cual le hizo contemplar las posibilidades que se abrían a investigaciones futuras. Había en ellas una potencialidad enorme para el avance del conocimiento de diversos problemas propios de la historia social y cultural.

Y con aquel doble magisterio, el de Caro Baroja y Charles Lea (cuyos trabajos sobre la brujería estaban fundamentados sobre las fuentes inquisitoriales), Gustav Henningsen se lanzó también a la búsqueda de los papeles del inquisidor Alonso de Salazar Frías, el conocido por sus detractores como abogado de las brujas. Se hace evidente en esto la aguda sensibilidad de Henningsen, lo que le permitió apreciar el valor de las fuentes documentales para el estudio de estos asuntos. Sin duda, su dilatada experiencia profesional en Dinamarca, donde trabajó en los archivos nacionales de su país ya desde sus años de estudiante universitario y también, después, desde su primer destino laboral, le llevó a cultivar una particular inclinación por el ejercicio propio del archivero. A este hecho debe de atribuirse, más que a la buena fortuna o al azar, el hallazgo que hizo entre 1967 y 1968 de la documentación que manejó Charles Lea para elaborar su capítulo sobre la intervención de la Inquisición en el problema de la brujería (Lea, 1907, vol. IV, cap. IX). Documentación que también manejó después, aunque de forma indirecta, Julio Caro Baroja cuando abordó el asunto de la brujería vasco-navarra de principios del siglo XVII, dedicándole a ello varios capítulos de su libro Las brujas y su mundo (1961) -un clásico en la materia desde su aparición-. Pero, ni Lea ni Caro Baroja habían manejado directamente estos fondos documentales. A Charles Lea le mandaron a Estados Unidos copias de parte de la documentación existente sobre el asunto, entonces depositada en el Archivo General de Simancas, en Valladolid. Y Caro Baroja había usado los trabajos de este autor como guía documental, a lo que añadió algunas otras fuentes, de extraordinaria riqueza, sin duda, pero al margen de los archivos de la Inquisición, salvo los documentos de la Biblioteca Nacional de Madrid que encontró entre sus fondos.

Como archivero que era, durante su paso por el Archivo Histórico Nacional de Madrid, Henningsen dio valor prioritario a la documentación inquisitorial que allí existía. En la primavera de 1967 dedicó dos meses a estudiar estos fondos con la intención de entender su particular organización y la naturaleza de las series que contenían. Contempló las muchas posibilidades que todo aquello ofrecía, e ideó hipótesis de trabajo que solo son posibles cuando se tiene un conocimiento suficiente de la disciplina archivística y de la composición y naturaleza de los materiales del archivo. Finalmente, se replanteó los métodos de investigación al uso a partir del cotejo entre los problemas teóricos que se venían planteando y los recursos disponibles para su estudio. Pueden ser muy distintas las reacciones que experimentan los estudiosos cuando entran en contacto directo con los materiales que fundamentan la construcción del conocimiento, en este caso, con los documentos del archivo. Y tal experiencia se suele manifestar de maneras muy diversas en cada uno. En el caso de Gustav Henningsen, su reacción estuvo determinada, 
sin duda, por la múltiple formación en la que él se había venido ejercitando: la propia del antropólogo, del historiador y del archivero.

Por un lado, ideó la creación de un instrumento para la investigación a partir del trabajo sistemático con la serie documental denominada «Relaciones de Causas»-de la que hablaremos a continuación-, herramienta que antes de concluirse comenzó ya a dar rendimientos importantes en su propia investigación y también en la de otros investigadores, sin que a veces sus beneficiarios, inmersos en trabajos iniciáticos o "doctorales», reconocieran de dónde procedía tal beneficio. Aquel era un «instrumento de archivo», como se dice en el argot archivístico, que era válido tanto para realizar análisis sincrónicos comparativos de distintos fenómenos de naturaleza social o cultural como análisis diacrónicos, dispuestos al examen de las evoluciones de tales fenómenos. Servía también, aunque fuera con propósitos menos ambiciosos, para establecer la demarcación de cuestiones muy concretas que se pretendieran someter a examen.

Por otro lado, una vez redescubrió la documentación inquisitorial relativa a la brujería vasco-navarra, perdida durante medio siglo, decidió concentrar su trabajo en ella. La riqueza de esta voluminosa documentación es extraordinaria e informa con todo detalle del problema tal como evolucionó a lo largo de las décadas iniciales del siglo XVII. De su importancia para el conocimiento del fenómeno dio cuenta de forma inmediata en el informe que publicó en 1969 en la revista nórdica Temenos, en el cual explicaba que en esos libros y legajos localizados en el archivo había mucho más, y de mayor importancia si cabe, de lo que manejó Charles Lea primero y Caro Baroja después. Henningsen tituló este trabajo «The papers of Alonso de Salazar Frías» y expresó su intención de dedicar su investigación al análisis completo de dicho material con el objetivo de cuestionar las tesis entonces debatidas sobre el fenómeno de la brujería y de la brujomanía.

La riqueza del material encontrado le permitiría alcanzar su propósito encaminándose en tres direcciones. Primero, siguiendo lo hecho por Julio Caro Baroja, avanzaría en el estudio biográfico del inquisidor Alonso de Salazar Frías, pues representaba sin duda un personaje paradigmático en la concepción moderna del problema de la brujería, ya que lo había hecho con un claro sesgo "científico». Además, las propuestas de este singular inquisidor habían tenido una influencia determinante en el gobierno de la Inquisición y, a consecuencia de ello, en la evolución de los acontecimientos en el territorio vasco-navarro y, siguiendo su ejemplo, en el resto de los territorios del imperio español. La segunda senda por la que se encaminó estuvo determinada por el hallazgo de uno de los ocho volúmenes que comprendía el libro de la visita que Salazar había realizado por toda la región en la que, durante casi ocho meses, hizo sus pesquisas. Este volumen, señalado por Salazar con la letra F, podía ser utilizado del mismo modo en el que el antropólogo contemporáneo reunía y analizaba su información. Y, por último, como tercera vía, los informes que el inquisidor Salazar fue elaborando y enviando a sus superiores en el Consejo de la Inquisición servían para establecer una evaluación cualificada de la naturaleza de las prácticas de brujería y, también, de las razones que provocaron la dimensión epidémica de estas creencias y miedos que suscitaron por regiones tan extensas. 


\section{EL ABOGADO DE LAS BRUJAS}

El estudio pormenorizado del caso vasco-navarro, a partir de unas fuentes de información de riqueza inusitada, servía para revisar teorías vigentes y muy extendidas sobre la brujería que ahora se hacían insostenibles. Resultaba inválida la idea de la existencia de grupos que practicaban la brujería como forma de manifestar una voluntad de resistencia o rebeldía, y que eran propias de las clases populares rurales enfrentadas a las élites procedentes del mundo urbano, acabando estos últimos por imponerse al aplicar la violencia en la represión de este tipo de prácticas. Tampoco se sostenía la interpretación propuesta con tanto éxito por la egiptóloga Margaret Murray, en la que la brujería manifestaba formas particulares de un mundo femenino y pagano, que estaban siendo reprimidas por parte del poder cristiano, encabezado por un orden masculino. Tales interpretaciones, con sus distintas variantes, daban crédito a la existencia de la brujería entre distintos grupos, como expresión de una u otra aspiración social. Pero ahora resultaban muy cuestionables todas estas ideas y no era fácil que se mantuvieran en pie tras confrontarlas con uno de los ejemplos mejor documentados para la historia de Europa, el cual fue desgranado por Henningsen con todo detalle.

Es cierto que tales creencias mágicas estuvieron muy extendidas en el pasado, estallando periódicamente en verdaderas oleadas de «epidemia onírica» y provocando, incluso, episodios de histeria colectiva y persecuciones incruentas. Pero la práctica brujeril, tal como era caracterizada por tales creencias, y tal como explicaban las distintas interpretaciones historiográficas, no había existido jamás. Si acaso hubo algo, apenas fueron casos individuales, aislados y muy marginales, comportamientos que podían tener algún punto en común con lo descrito, pero poco más. Tal vez esta sea la aportación más valiosa del libro que Gustav Henningsen, compuesto tras su hallazgo de aquel material precioso en los archivos de Madrid y que, tras diez años de espera, apareció publicado en inglés y en Estados Unidos de América con el título, que traducimos al español, El abogado de las brujas. Brujería vasca e Inquisición española (Henningsen, 1980).

Es muy probable que la verdad científica mostrada por Gustav Henningsen, tal como ya le ocurriera a la verdad inquisitorial demostrada a principios del siglo XVII por su biografiado, Alonso de Salazar Frías, no consiga hacer rectificar las creencias actuales cuando se asoman a este asunto. Hoy, por ejemplo, cuando se viaja a Zugarramurdi -centro simbólico de la brujería- lo que escuchamos es una nueva interpretación que, por sorprendente que pueda parecer, va cobrando cada día mayor fuerza, y que sin duda acabará fundiendo las interpretaciones anteriores en una inédita, esta de cuño nacionalista, que vuelve a afirmar la existencia de tales prácticas y grupos de brujos. La brujería vasca fue -se nos dice- una expresión de resistencia en las tradiciones paganas que vivían insertas en el pueblo vasco, donde el protagonismo femenino siempre había sido principal, y que se vieron amenazadas por los poderes extraños que desde el interior peninsular intentaron someter a este pueblo milenario, haciendo uso para ello de la fe cristiana y de las armas de la Inquisición. El subtítulo que Henningsen dio a su libro Brujería vasca e Inquisición española, habrá quien lo interprete en este mismo sentido. Aunque lo cierto es que tal expresión respondía a las particulares circunstancias editoriales de los años de la 
década de 1970 que asistieron al alumbramiento del libro en la ciudad de Reno. William Douglass, el editor norteamericano de la obra, y a le vez antropólogo de la Universidad de Nevada, colaboraba entonces con los Laxalt, familia descendiente de inmigrantes vascos, en la creación de un centro de estudios vascos en esa Universidad, y que estuvo estrechamente conectado con la editorial universitaria ${ }^{1}$. En este caso, el subtítulo de su obra se ajustó más al propósito de promocionar los estudios vascos en Norteamérica que a la interpretación que en el siglo XXI se podrá hacer de la brujería.

Aunque en el libro se dice una y otra vez que el tema tratado es el de la «brujería vasca» -lo que sin duda debe de obedecer a estas mismas razones impuestas por los fundadores de la editorial norteamericana-, en realidad el fenómeno estudiado no se reduce a este ámbito geográfico, sino que aborda un espacio territorial mayor, que se extiende por Navarra, La Rioja y el norte de Burgos. De hecho, el principal brote del fenómeno que se analiza en el libro tuvo lugar en los valles pirenaicos del noroeste de Navarra, en especial en el valle de Baztan, desde donde se extendió posteriormente a localidades de Guipúzcoa y Vizcaya. Por otro lado, cuando Gustav Henningsen se decidió a estudiar la brujería en este escenario geográfico adoptando para ello una perspectiva histórica, lo hizo con el objetivo de realizar una comparación entre lo ocurrido a ambos lados del Pirineo: al norte, en las tierras francesas del País de Labourd, y al sur, en las tierras vascongadas y navarras.

Pero, a pesar de esta disposición hacia la historia comparativa, estamos en verdad ante uno de los trabajos pioneros en la llamada «microhistoria». Y en este intento, la luz arrojada por el trabajo de Gustav Henningsen está llamada a perdurar, ya que los conocimientos conseguidos a partir de su análisis «micro» afectan a cuestiones generales o a "macrorrealidades». Y lo hace como pocos trabajos de la llamada «microhistoria» lo han conseguido hacer, pues consigue mostrar con gran evidencia la lógica de problemas generales de la larga duración histórica a partir de un análisis profundo en el breve espacio temporal y geográfico. Con esta perspectiva, la cuestión fundamental que plantea su investigación, aunque quede en el fondo del problema, fue explicar qué razones hicieron que al norte de los Pirineos las autoridades combatieran el miedo a la brujería aplicando el fuego con una violencia feroz y al sur, muy al contrario, lo hicieran aplicando la razón científica.

\section{ALONSO DE SALAZAR FRÍAS, INQUISIDOR Y HUMANISTA}

La tesis que ha sostenido Gustav Henningsen se muestra en la primera página de su libro El abogado de las brujas, en las líneas de su dedicatoria. Y aunque haya podido pasar desapercibida por lo breve de su expresión, es enormemente provocadora. Toda-

1 El libro apareció así: Gustav Henningsen, The Witches' Advocate. Basque Witchacraft and the Spanish Inquisition, Reno, Universitity of Nevada Press, 1980. Esta editorial universitaria, nacida en 1961, fue fundada por Robert Laxalt, y el centro de estudios vascos (Center for Basque Studies) creado en esta misma universidad fue fundado también por él unos años más tarde, en 1967. William Douglass, que colaboró estrechamente con Robert Laxalt en esta empresa, había conocido a Gustav Henningsen en Bera de Bidasoa, en la casa de Julio Caro Baroja, en 1964. Henningsen recuerda este encuentro así: «Durante mi estancia en Bera, conocí brevemente a otro de los apadrinados por don Julio: un joven antropólogo norteamericano, William Douglass. Este encuentro resultó, años más tarde, ser de mucho provecho para mi» (Henningsen, 2010, p. 428). 
vía, cuando hoy se lee, sigue despertando cierta perplejidad a poco que se piense en ello. No sería exagerado decir que ningún historiador español de aquel tiempo, ni tal vez de hoy, se hubiera atrevido a hacer algo parecido. Y es que Henningsen dedica su obra «a la memoria de D. Alonso de Salazar Frías, inquisidor y humanista español». Pero, ¿cómo un historiador puede dedicar su obra principal a un inquisidor español? Y, en segundo lugar, ¿cómo un inquisidor puede ser a la vez juez de la Inquisición y humanista? Dirigirse así, de entrada, a un lector de finales del siglo XX o de principios del siglo XXI, solo resulta posible con un trabajo de investigación contundente, apoyado sobre unas bases documentales incontestables, y razonando de manera que quede poco espacio abierto a la especulación y a la polémica. Alonso de Salazar (inquisidor, sacerdote y hombre de leyes) se mostró en su intervención sobre el problema de la brujería como un verdadero humanista y aplicó métodos que podrían calificarse de científicos en su esfuerzo por discernir sobre la realidad del asunto. Tras el análisis de las reflexiones del inquisidor y de los consejos dados al gobierno inquisitorial, Charles Lea, Geoffrey Parrinder y Caro Baroja ya habían señalado la modernidad de este inquisidor ${ }^{2}$. No eran pocos los estudiosos que habían reconocido abiertamente su admiración por sus razonamientos y, entre ellos, algunos incluso le habían dedicado anteriormente sus publicaciones. Era el caso de Charles Williams, quien dedicó su libro Witchcraft (1941) a «la inmortal memoria de Alonso Salazar» (Henningsen, 1978, p. 582).

Pero el análisis moderno -científico- que el inquisidor había realizado sobre la brujería y la brujomanía, y que había vertido en unos memoriales ya conocidos y estudiados brevemente por Lea, alcanzaba ahora un desarrollo mucho más revelador, en el método seguido y en las conclusiones obtenidas. Y ello ha sido merced a la investigación novedosa y paciente de Henningsen, que abordó la visita inquisitorial realizada por Salazar a lo largo de casi ocho meses por las poblaciones afectadas por la brujomanía, verdadera epidemia llegada desde el norte de los Pirineos hacia 1600. La visita, práctica inquisitorial ordinaria que resultó fundamental para entender lo que Jean Pierre Dedieu ha descrito como la «inserción social» de la institución, precisa todavía de estudios que muestren el sentido y valor cabal de su incidencia sobre la realidad local (Dedieu, 2004). Y ente este sentido, el estudio que hace Henningsen de la visita de Salazar de 1611 es un modelo de análisis magistral. El mencionado hallazgo, a finales de 1967, de uno de los ocho volúmenes del libro resultante de dicha visita le permitió a Henningsen reconstruir las informaciones de los siete volúmenes perdidos: acervo documental que en total tendría más de 5600 folios y que recogía datos sobre unas dos mil personas que confesaron, en un primer momento, pertenecer a la secta brujeril y participar de todas aquellas prácticas que se les achacaba.

El procedimiento seguido por Salazar en su visita no estaba lejos de los métodos que utilizan los antropólogos en sus trabajos de campo. Salvando las distancias, y con más de

2 «En mi caso, fue la lectura del libro de Geoffrey Parrinder, de 1958, sobre brujería europea y africana, lo que me puso sobre la pista de los papeles de Salazar. Los menciona en una sola página, pero fue para mí lo suficiente como para comprender las posibilidades que encerraría aquel material. Al parecer ese escéptico inquisidor, que actuó a principio del siglo XVII, había llevado a cabo un trabajo de campo a la moderna y, como algo insólito para su tiempo, había llegado a dudar de la existencia de las brujas» (Henningsen, 2010, p. 429). 
tres siglos de diferencia, tampoco eran muy distintos los propósitos del inquisidor y los de quienes han cultivado esta disciplina del conocimiento. El objetivo de Salazar era conocer la realidad de tales fenómenos y explicar sus mecanismos. De tal manera, Henningsen, metido en la piel del inquisidor Salazar, podría penetrar en las pequeñas sociedades locales de principios del siglo XVII para descubrir en ellas los detalles, de otra manera irrecuperables, de sus prácticas y creencias mágicas. Y tras seguir minuciosamente el trabajo del inquisidor, analizando lo que había y reconstruyendo lo perdido, era posible ahora entender mejor las conclusiones que alcanzó. Se podría comprender el razonamiento de su descreimiento en la existencia generalizada de sectas de brujos, su escepticismo crítico, y, en consecuencia, los consejos que dio al gobierno de la Inquisición sobre cómo se debía actuar ante este fenómeno para asegurar el restablecimiento de la paz social. La Inquisición, además, debía exigir la exclusividad jurisdiccional sobre este asunto e impedir la intervención de las justicias civiles. Y una vez conseguido esto, para erradicar la brujería, debía imponerse el silencio sobre el asunto. «No hubo brujos ni embrujados en el lugar hasta que se comenzó a tratar y escribir de ellos (El inquisidor Alonso de Salazar Frías, 1612)», con esta lacónica conclusión arranca el libro El abogado de las brujas (Henningsen, 2010).

En su esfuerzo por comparar la evolución del problema en distintas áreas geográficas y discernir por qué en buena parte de Europa se siguieron quemando brujas mientras esto se dejó de hacer en los territorios de la monarquía española (incluidas las tierras italianas y americanas), Henningsen atribuye a Salazar un mérito fundamental. Tras su examen empírico y su observación de la realidad, el inquisidor concluyó que todo aquello obedecía a diversas razones que explicó de manera convincente, las cuales son hoy tema de estudio de las ciencias sociales, ya sea la sociología o la psicología social. Pero Henningsen demostró algo más: Salazar no era una excepción en su tiempo, sino que existía una corriente bastante nutrida de escépticos entre los pensadores y también entre los ministros del rey. Tampoco faltaban entre los miembros que integraban el gobierno de la Inquisición, en sus tribunales de distrito y en el Consejo de la Suprema, en la corte del rey.

Una vez el gobierno de la Inquisición quedó convencido de la irrealidad del fenómeno, tal como se imaginaba entonces, se impuso a los tribunales de distrito una forma particular de intervenir en estos casos con la que se asegurara la disolución del problema. La Inquisición renunciaba al fuego y prefería formas más sutiles y benignas en su lucha contra la herejía. Y para evitar que su estrategia disuasoria fuera contrariada, se aseguró que no actuaran sobre estos asuntos las justicias civiles, partidarias de aplicar el rigor extremo para satisfacer con ello la presión social. Y así se pudo hacer por ser la Inquisición como era. El poder de la Inquisición en la corte y su particular estructura organizativa, bien jerarquizada y extendida por toda la geografía, facilitaron que el nuevo modelo de intervención sobre este problema se impusiera definitivamente. Y con ello, pese a que las creencias en la brujería siguieron existiendo en la sociedad, la actuación inquisitorial en los territorios de la monarquía española aseguró que no se repitieran los episodios recurrentes de epidemias oníricas, los cuales venían acompañados del miedo colectivo y de la quema de numerosos brujos y brujas para combatirlo.

El examen comparativo que hizo Henningsen del fenómeno de la brujería, que aparecía de forma simultánea en distintos lugares, pero evolucionando de manera tan dispar, 
nos muestra lecciones importantes. En el sur de Francia (País de Labourd), las justicias civiles condenaron a la muerte a miles de personas y, por el contrario, en el norte de España se optó por una intervención inquisitorial conducida por criterios racionales. La explicación de esta disparidad nos lleva a preguntarnos de qué manera la intervención de la justicia y de la política conformó las sociedades y la cultura europea en la temprana modernidad. Y, en este sentido, el análisis de los hechos históricos obliga a cuestionarse muchos de los prejuicios que todavía están vigentes en la interpretación que de nuestro pasado han ofrecido los historiadores. Adriano Prosperi advertía de esta circunstancia en unas reflexiones acerca de la historia de la Inquisición italiana, cuando se preguntaba por la razón que había llevado a los intelectuales de su país a no prestar la atención que merecía la obra de fray Leandro Alberti en la construcción de la Italia moderna. Su condición de fraile dominico y de inquisidor no tenía por qué contradecir que hubiera sido uno de los principales precursores en el siglo XVI de la concepción unitaria de la nación italiana, que terminaría por consolidarse en el siglo XIX (Prosperi, 2006). Al margen de la vieja y larguísima polémica entre los detractores y defensores de la Inquisición, la aportación de la obra de Gustav Henningsen se encaminó por la senda de análisis desprejuiciado, demostrando con ello la potencialidad de esta perspectiva para el avance de nuestro conocimiento.

\section{DE LAS FUENTES Y MÉTODOS DE ANÁLISIS EN LOS ESTUDIOS INQUISITORIALES}

Sin duda, en lo referido a los estudios inquisitoriales, la aportación que Gustav Henningsen hizo en el tema de la brujería resulta paradigmática todavía hoy, pues su modelo de estudio sobre el inquisidor Alonso de Salazar Frías sigue siendo vigente. Sin embargo, sus aportaciones en lo referido a los archivos de la Inquisición española han tenido, si cabe, un valor todavía mayor, pues los trabajos que ha realizado en este campo han legado a los investigadores conocimientos de enorme utilidad sobre estas fuentes documentales y sobre su posible aprovechamiento.

En un principio, su interés por los fondos documentales de la Inquisición nació de la necesidad de encontrar informaciones acerca de las prácticas y creencias de los gallegos en el pasado, para ponerlas en relación con las que había reunido en las grabaciones realizadas en sus estudios de campo en los años sesenta. Pero una vez estuvo entre los papeles de la Inquisición, su experiencia profesional como archivero le hizo utilizarlos en un sentido mucho más amplio. Desentrañó la lógica de estos fondos, tal y como habían quedado organizados a su llegada a principios del siglo XX a las dependencias del Archivo Histórico Nacional. Pudo entender cuál fue su primera organización y evaluar la proporción de lo que todavía existía después de tanta destrucción. Se preguntó por el modo en el que actuaron los archiveros del Santo Oficio y por el tipo de documentación que produjo la institución a lo largo de su historia. En fin, conforme reunía documentación sobre la brujería y hechicería gallega, y según avanzaba en su búsqueda de los papeles del inquisidor Salazar, en sus cuadernos de notas reconstruyó el conjunto de los archivos de la Inquisición, tal como habían existido en sus días. Y, a la par, delineó el perfil de los restos que todavía quedaban, evaluando 
además cuáles eran sus contenidos precisos. Es cierto que sobre esto ya habían escrito algunos historiadores antes que él, pero había sido de forma parcial y poco segura. Y, por otra parte, los esfuerzos de los archiveros estatales que manejaban el enorme fondo documental, tarea que ha sido imprescindible para su utilización posterior, no estaban orientados a la especulación sobre las posibles líneas de investigación que podrían impulsar.

Hacia 1970, Gustav Henningsen había dado con dos claves fundamentales para la comprensión de los fondos inquisitoriales y de las posibilidades que ofrecían. En primer lugar, demostró que la mayor parte de lo que existía era el archivo particular del Consejo de la Suprema Inquisición, y que este se conservaba casi en su integridad. Poco de él se había destruido o desaparecido. En segundo lugar, dentro de estos fondos existía una serie documental, las «Relaciones de Causas», que permitían reconstruir fielmente la actividad procesal que los 21 tribunales de distrito llevaron a cabo durante los primeros dos siglos de su historia. Por lo tanto, pese a la destrucción de la mayor parte de los archivos de los tribunales de distrito, se podía conocer la totalidad de los procesos que estos habían llevado a cabo desde mediados del siglo XVI hasta 1700 . Y, en consecuencia, era posible reconstruir su historia con suficiente exactitud, al menos la de su actividad en la lucha contra las distintas expresiones de la herejía.

Henningsen calificó a la serie formada por las relaciones de causas como el «eje principal del archivo» del Consejo de la Suprema, el cual resultaba extraordinariamente revelador. Si en algo destacó la modernidad de la institución inquisitorial fue, sin duda, en la producción, ordenación y gestión de la información. Como institución bien jerarquizada y centralizada que fue, el Consejo de la Inquisición que la presidía, órgano de gobierno y de control sobre los tribunales de distrito, reunía en sus archivos la información fundamental de todos ellos. Y la guardaba de manera muy eficiente, con criterios archivísticos modernos que se anticiparon a lo que sería la Archivística contemporánea, aunque acomodados a la lógica propia de aquellos tiempos y a las prioridades que marcaban su función en la monarquía. Henningsen entendió que toda aquella vasta información que se producía en los terminales de distrito, y que se archivaba en la cabecera de la Inquisición, tenía una función práctica: eran la memoria y el nervio vivo de la institución. La circulación constante de tal información, desde la cabeza hasta sus miembros y viceversa, hacían posible el funcionamiento eficiente de todo el cuerpo institucional, el cual se extendía por el conjunto de la monarquía. Esta era la clave que garantizaba la consecución de sus propósitos.

«Seis generaciones de inquisidores» a lo largo de los siglos XVI y XVII se habían implicado en la producción, circulación y custodia de tanta información. Y con los inquisidores, una gran cantidad de colaborares trabajaron en este mismo cometido. La idea que presidió esta empresa fue inquirir en la realidad todo lo posible y dejar tras ello un rastro informativo suficiente que pudiera ser recuperado de manera segura y rápida en el caso de necesitarse. Información que además podía ser compartida por todos los tribunales gracias al control y coordinación que el Consejo ejercía sobre ella. Por esta razón Henningsen hablaba de los archivos de la Inquisición como un «gran banco de datos». Y era tal la dimensión de aquel banco que tuvo que idearse 
una herramienta para su manejo. Esta era la principal utilidad de las «Relaciones de Causas», registro periódico que cada tribunal enviaba de su actividad procesal al Consejo y que este guardaba en una serie específica con este nombre. Henningsen fue el primer historiador que de manera sistemática se propuso cuantificar y evaluar el conjunto total de la serie.

Si aquella herramienta había sido útil para los inquisidores cuando se preguntaron por distintas cuestiones concernientes a su oficio, lo mismo debería de ser para los investigadores actuales. Podían cambiar las preguntas, y sobre todo las intenciones que había tras ellas, pero el instrumento utilizado para dar con las respuestas era el mismo. Solo era necesario reconstruir aquella maquinaria y volver a reunir todas sus piezas para ponerla en funcionamiento. Este fue el proyecto que Gustav Henningsen fue elaborando en su cabeza conforme desentrañaba la lógica de los archivos de la Inquisición a finales de la década de los años sesenta, hasta que, llegado el otoño de 1971, se puso manos a la obra para desarrollarlo. En los ensayos parciales que habían realizado antes que él investigadores como Henry Charles Lea, Toribio Medina (para los tribuales americanos) o Schäfer (para el protestantismo en España en el siglo XVI) se había demostrado que las posibilidades de esta serie documental -las «Relaciones de Causas»- eran bien ciertas. Pero todo aquello era demasiado antiguo, de finales del siglo XIX y de los años muy iniciales del siglo XX, cuando los archivos de la Inquisición estaban todavía en Simancas (Valladolid). Sus propias indagaciones entre 1967 y 1968 en los archivos de Madrid sobre la brujería y hechicería gallega le convencieron de que era posible hacerlo ahora, incluso con objetivos mucho más ambiciosos, no renunciando a completar la totalidad del trabajo posible.

Así, Gustav Henningsen se entregó a esta tarea cuando tenía treinta y cinco años de edad y en España se iba acabando la dictadura de Franco. Se entiende el empuje optimista aplicado en este proyecto, en el cual trabajó de manera sistemática entre 1972 y 1978, culminando una primera etapa en la que consiguió, en pocos años, un avance muy significativo. Sus conclusiones pudieron escucharse en distintos congresos tanto nacionales como internacionales entre 1976 y los primeros años ochenta, lo cual significó una verdadera convocatoria general para que los investigadores comprobasen la eficacia de sus logros. En un texto publicado en el Boletín de la Real Academia de la Historia en 1977 daba las señas básicas de su trabajo: "El "banco de datos" del Santo Oficio: las relaciones de causas de la Inquisición española» (Henningsen, 1977). A modo de ejemplo, mostró a través del Tribunal de Sicilia las posibilidades que su método ofrecía para reconstruir su historia y su actividad procesal. El archivo de este tribunal italiano fue quemado públicamente a finales del siglo XVIII en Palermo. Pero con las relaciones de causas, que se habían enviado periódicamente desde la isla hasta Madrid entre 1547 y 1701, y que estaban guardadas en el archivo del Consejo, era posible ahora reconstruir lo desaparecido. Y hacerlo no suponía mucho tiempo de trabajo, aunque lo hiciera un solo investigador. Para el caso siciliano había un total de 115 relaciones de causas encuadernadas en cinco tomos, y en ellas se recogían los 3188 procesos de fe que incoó el tribunal italiano en los siglos XVI y XVII. A partir de esto, la investigación podría avanzar por sendas insospechadas, y con toda seguridad, inalcanzables de otro modo. 
Henningsen calculó que en el archivo del Consejo radicado en Madrid el total de las relaciones de causa alcanzarían unos cien tomos con un promedio de mil páginas cada uno. Mucho, es cierto, pero abarcable por el trabajo en equipo, y realizable en no demasiados años. Era consciente de las pérdidas de algunas de estas relaciones de causas y de que otras podrían aparecer en diversos fondos españoles o extranjeros. Tampoco ignoraba el escaso rigor de algunos de los ministros de la Inquisición al archivar estos registros. Pero descontando todo esto, los resultados que se podrían alcanzar, ya en el primer intento del proyecto, serían muy importantes. Y, en cualquier caso, todas aquellas lagunas, que significaban una proporción pequeña frente al conjunto, podrían ser cubiertas posteriormente hasta completar el cuadro en su integridad. El tema era suficientemente conocido en el ámbito internacional y el interés despertado estaba tan extendido que no faltaría la colaboración de la comunidad científica en su conjunto (historiadores y archiveros) para que se pudiese culminar el proyecto.

El clima existente en la investigación española e internacional en los años setenta invitaba a esto. Y los trabajos sobre la Inquisición realizados y publicados en los ochenta confirmaron la utilidad del proyecto realizado por Henningsen. Los estudios monográficos sobre cada uno de los tribunales necesitaban de esta serie documental como guía principal para establecer los perfiles concretos de la actividad procesal que desarrollaron. Incluso, la comparación entre los distintos tribunales de distrito y entre las actividades procesales que acometieron tenían que partir del uso de esta herramienta. Si acaso, el asunto de estudio elegido era una creencia o comportamiento herético en particular, las relaciones de causa también se hacían imprescindibles. El mismo Henningsen, por ejemplo, nos ha dejado una geografía general de la brujería, de la hechicería y de prácticas de "magia blanca» haciendo uso de este material, de su método y herramienta. Dicha geografía nos muestra todo el territorio peninsular, el continente americano y el sur de Italia, y pone a la vista las grandes corrientes, diferenciadas, de creencias y prácticas propias de la cultura popular en la larga duración.

La «cartoteca» elaborada por Henningsen -eran tiempo en los que la informática no se había impuesto todavía - valía para conducir cualquiera de estas líneas de investigación y señalar cuáles eran los aspectos principales a analizar. Así lo han reconocido muchos investigadores en las páginas de sus publicaciones que fueron apareciendo en aquellos años, menos de los que en realidad se valieron de ellas. Y cuando no se utilizaban directamente las tarjetas de su «cartoteca», se componían las propias, pero siguiendo su experiencia. El valor de esta fuente, como quedó demostrado, era tan universal que nadie que entrara en este campo de estudios podría pasarla por alto. En los territorios de Historia social o cultural ofrecía extraordinarias oportunidades. Se justifica así la insistencia de Henningsen cuando de manera continuada ha llamado la atención sobre ello. Y se comprenden también sus reflexiones tiempo después, cuando en Alemania, en un congreso organizado por el Instituto Max-Planck se lamentaba por las oportunidades perdidas (Henningsen, 1993). No es este el lugar para entrar en ello. Creo, no obstante, que más temprano que tarde, se acabará por hacer lo que él propuso insistentemente y que dejó en un punto muy avanzado de ejecución. Será, sin duda, para beneficio de la comunidad científica. 


\section{LISTA DE REFERENCIAS}

Caro Baroja, J. (1961). Las brujas y su mundo. Madrid: Revista de Occidente.

Dedieu, J. P. (2004). De la Inquisición y su inserción social. Nuevas directrices en la historiografía inquisitorial. Coloquios de Historia Canario Americana, XVI (pp. 2116-2129) Las Palmas: Cabildo de Gran Canaria.

Henningsen, G. (1977). El «banco de datos» del Santo Oficio. Las relaciones de causa de la Inquisición española (1500-1700). Boletín de la Real Academia de la Historia, 174, 547-570.

Henningsen, G. (1980). The Witches' Advocate. Basque Witchacraft and the Spanish Inquisition. Reno: Universitity of Nevada.

Henningsen, G. (1993). The Database of the Spanish Inquisition. The relaciones de causas-project revisited. En Heinz Mohnhaupt y Dieter Simon (dirs.), Vorträge zur Justizforschung. Geschichte und Theorie (pp. 43-85). Frankfurt am Main: Max-Planck-Institut für Europäische Rechtsgeschichte.

Henningsen, G. (2010). Andanzas por España de un inquisidor a la moderna de raza nórdica. Huarte de San Juan, 17, 423-441.

Lea, H. Ch. (1906-1907). History of the Inquisition of Spain. 4 vols. Nueva York-Londres: Macmillan. (Disponible online en The Library of Iberian Resources).

Prosperi, A. (2006). Nuove prospettive per una storia dell'Inquisizione. Cromohs, 11, 1-6. 
0 